Proyecciones Journal of Mathematics

Vol. 38, No 1, pp. 129-144, March 2019.

Universidad Católica del Norte

Antofagasta - Chile

\title{
Codes detecting, locating and correcting random errors occurring in multiple sub-blocks
}

\author{
Pankaj Kumar Das \\ Tezpur University, India \\ Received: March 2018. Accepted : August 2018
}

\begin{abstract}
In this paper, we study bounds on the number of check digits of linear codes that can detect multiple sub-blocks each affected by e or less random errors and can locate such corrupted multiple sub-blocks. Further, we obtain an upper bound on the number of check digits of linear codes which can correct such errors occurring in multiple subblocks. We also give examples of such codes.
\end{abstract}

Subjclass [2010] . 94B05, 94B65.

Keywords : Syndromes, Parity check digits, Bounds, Error locating codes 


\section{Introduction}

With the appearance of the paper entitled "Error-Locating Codes-A New Concept in Error Control" by Wolf and Elspas [17], a new direction of research in coding theory has been opened up and many papers are written in this direction. Some of them may be mentioned as [1], [2], [4], [5], [12], [15], [16]. The paper [17] introduced the concept of error-locating codes (EL-codes)-which stands in between error detection and error correction concept. EL-Codes are found to be useful for diagnosing fault in computer systems and reducing the retransmission cost in communication systems [11], semiconductor memory module [9]. In this technique, the block of received digits is subdivided into some mutually exclusive sub-blocks and while decoding, if error occurs within sub-block(s), one can detect the error as well as one can identify which particular sub-block(s) contains the error. Then retransmission of the corrupted sub-block(s) is sufficient instead of the whole block. But the study of the paper [17] is confined to binary codes and to single corrupted sub-block only, this paper takes the case of binary as well as non-binary and multiple corrupted sub-blocks. It is worth mentioning that location of several corrupted sub-blocks has been studied in $[2],[3],[12],[15],[16]$. In [12], the study was done with respect to a new metric. The paper [3] studies error-location codes which can locate mutiple sub-blocks with burst error and in [2], [15], [16], error-location codes are constructed by taking the tensor product of two codes. But the study is not done with respect to bounds on the number of parity check digits of a linear code that can locate several sub-blocks having random errors. The present paper is an attempt in this direction. We further extend the study to obtain bounds on the number of parity check digits of a linear code that can correct random errors in multiple sub-blocks. For similar works on blockwise correction of errors confined to a single sub-block, one may refer to $[6],[8]$.

The study of bounds on the number of parity check digits for a linear code is important in order to know the efficiency of the code. If the number of parity check symbols in a code is lesser, then the rate of information of the code will be higher. In fact, the ultimate capabilities and limitation of codes can be known by the bounds.

Now, we consider a linear code $C$ which is nothing but a proper subspace of $n$-tuples over $G F(q)$. If $G$ is a $k \times n$ generator matrix of the code $C$, 
then the number of information digits and parity check digits are $k$ and $n-k$ respectively. A matrix $H$ whose rows generate the null space of the code $C$ is known as the parity check matrix of the code $C$. The quantity $v H^{T}$ is called the syndrome of the vector $v$. The linear code of length $n$ digits with dimension $k$, consisting of $k$ information digits and $r=n-k$ parity digits, is divided into $m$ mutually exclusive sub-blocks where each sub-block contains $t=n / m$ digits. The rate of information of an $(n, k)$ code is $k / n$.

We assume an $(n, k)$ linear code over $G F(q)$ which is divided into $m$ mutually exclusive sub-blocks and the number of corrupted sub-blocks is at most $l$, each sub-block is affected by $e$ or less errors where $l \leq m$. Now we state three conditions as below.

(i) The syndrome due to errors in any $l$ or less sub-blocks, each sub-block affected by at most $e$ errors, must be non-zero.

(ii) The syndrome due to errors in any $l$ or less sub-blocks, each sub-block affected by at most $e$ errors, must be distinct from the syndrome resulting from such errors confined to any other set of $l$ or less subblocks.

(iii) The syndrome due to errors in any $l$ or less sub-blocks, each sub-block affected by at most $e$ errors, must be distinct from the syndrome resulting from such errors occurring in the same set of $l$ or less subblocks.

A linear code capable of detecting any $l(\leq m)$ corrupted sub-blocks each sub-block affected by at most $e$ errors must satisfy the condition $(i)$. A linear code capable of locating any such $l(\leq m)$ corrupted sub-blocks must satisfy the condition $(i)$ and $(i i)$. A linear code which is capable of correcting all such errors must satisfy all the three conditions $(i),(i i)$ and (iii).

The paper is arranged as follows. Section 1 is the introduction where we discuss briefly about the basic idea and importance of the study. Section 2 obtains lower and upper bounds on the number of parity check digits of a linear code that can detect any $l$ or less corrupted sub-blocks, each corrupted by $e$ or less errors. Section 3 obtains lower and upper bounds on the number of parity check digits of a linear code that is capable of locating any such $l$ or less corrupted sub-blocks. In Section 4, we obtain similar bounds for a linear code that can correct such $e$ or less errors affecting $l$ or less sub-blocks. 


\section{Detection of $e$ or less errors in multiple sub-blocks}

This section derives lower and upper bounds on the number of check digits of a linear code over $G F(q)$ detecting multiple corrupted sub-blocks each containing $e$ or less errors.

Theorem 2.1. An $(n, k)$ linear code over $G F(q)$ which is divided into $m$ mutually exclusive sub-blocks of length $t$ each, is capable of detecting any $l(\leq m)$ or less corrupted sub-blocks each containing e or less errors. The number of parity check digits $r$ needed for such an EL-code is given by

$$
r \geq \log _{q}\left[1+\sum_{j=1}^{l}\left\{\sum_{i=1}^{\lfloor e / 2\rfloor}\left(\begin{array}{l}
t \\
i
\end{array}\right)(q-1)^{i}\right\}^{j}\right] .
$$

( $\lfloor x\rfloor$ means the greatest integer less than or equal to $x$.)

Proof. In the proof, we first count the number of distinct syndromes according to conditions $(i)$ and then take this number less than or equal to $q^{r}$, maximum possible number of distinct syndromes.

For any $j \leq l$, any syndrome produced by $j\lfloor e / 2\rfloor$ or less errors occurring in any $j$ sub-blocks with a sub-block containing not more than $\lfloor e / 2\rfloor$ errors must be distinct from any such syndrome likewise resulting from such $j\lfloor e / 2\rfloor$ or less errors in the same set of $j$ sub-blocks. Otherwise we get a combination of at most $e$ errors occurring in any $j$ sub-blocks resulting in the zero syndrome, which can not be true by condition $(i)$. Since there are $\left(\begin{array}{l}t \\ i\end{array}\right)$ combinations of $i$ errors in $t$ components in a given sub-block, the number of such syndromes out of any $j$ sub-blocks is

$$
\left\{\sum_{i=1}^{\lfloor e / 2\rfloor}\left(\begin{array}{l}
t \\
i
\end{array}\right)(q-1)^{i}\right\}^{j}
$$

Therefore, there are

$$
\sum_{j=1}^{l}\left\{\sum_{i=1}^{\lfloor e / 2\rfloor}\left(\begin{array}{l}
t \\
i
\end{array}\right)(q-1)^{i}\right\}^{j}
$$

distinct syndromes, excluding the all zeros syndrome. Thus, we get 


$$
\left.q^{r} \geq 1+\sum_{j=1}^{l}\left\{\sum_{i=1}^{\lfloor e / 2\rfloor}\left(\begin{array}{l}
t \\
i
\end{array}\right)(q-1)^{i}\right\}^{j}\right]
$$

The following result provides an upper bound on the number of check digits required for the existence of such a code is derived. The proof of the result follows the technique of Varshamov-Gilbert-Sacks bound (refer Sacks [14] and Theorem 4.17 of Peterson and Weldon [13]). This technique gives us a method for construction of such codes in addition to the proof of the result.

Theorem 2.2. An $(n, k)$ linear EL-code over $G F(q)$ which is divided into $m$ mutually exclusive sub-blocks of length $t$ each, is capable of detecting any $l(\leq m / 2)$ or less corrupted sub-blocks each containing $e$ or less errors. There shall always exist such an EL-code having $r$ parity check digits provided that

$$
q^{r}>\sum_{i=0}^{e-1}\left(\begin{array}{c}
t-1 \\
i
\end{array}\right)(q-1)^{i} \times \sum_{s=0}^{l-1}\left(\begin{array}{c}
m-1 \\
s
\end{array}\right)\left(\sum_{i=1}^{e}\left(\begin{array}{l}
t \\
i
\end{array}\right)(q-1)^{i}\right)^{s} .
$$

Proof. By constructing a suitable $(n-k) \times n$ parity check matrix $H$ of the desired code, we prove the theorem. Assume that the columns of the first $m-1$ sub-blocks of $H$ and the first $j-1$ columns $h_{1}, h_{2}, \ldots, h_{j-1}$ of the $m^{\text {th }}$ sub-block have been added appropriately first and then we put down the conditions to add $j^{\text {th }}$ column $h_{j}$ of the $m^{\text {th }}$ sub-block of the matrix $H$ as follows:

According to condition $(i)$, the syndrome resulting from errors in any $l$ or less sub-blocks, each sub-block affected by $e$ or less errors must be nonzero. So, $h_{j}$ should not be a linear combination of previous any $e-1$ or less columns of the $m^{\text {th }}$ sub-block, together with any $(l-1) e$ or less columns among the previous any $l-1$ or less sub-blocks having not more than $e$ columns within a sub-block. In other words 


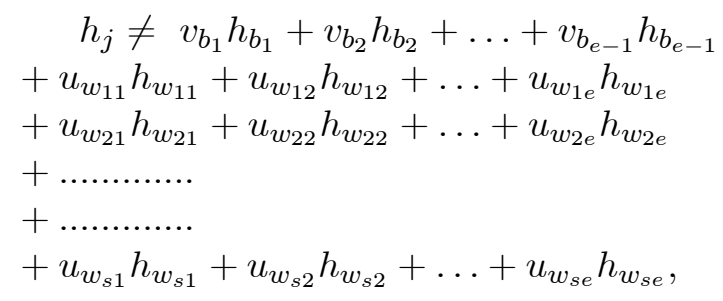

where $v_{b_{j}}, u_{w_{i j}} \in G F(q), s \leq l-1$ and $h_{b_{j}}$ 's are any $e-1$ columns from amongst the columns $h_{1}, h_{2}, \ldots, h_{j-1}$ of the $m^{\text {th }}$ sub-block and $h_{w_{i j}}$ 's are any $e$ columns within a sub-block (for same value of $i$ ) chosen from previous $m-1$ sub-blocks.

If the code satisfies this condition, the detection of $e$ or less errors each set occurring in any $l$ or less sub-blocks is ensured.

The linear combinations on the R.H.S. of (2.3), including the zero vector, can be counted as

$$
\sum_{i=0}^{e-1}\left(\begin{array}{c}
j-1 \\
i
\end{array}\right)(q-1)^{i} \times \sum_{s=0}^{l-1}\left(\begin{array}{c}
m-1 \\
s
\end{array}\right)\left\{\sum_{i=1}^{e}\left(\begin{array}{l}
t \\
i
\end{array}\right)(q-1)^{i}\right\}^{s}
$$

Therefore, we can add the column $h_{j}$ to the $m^{\text {th }}$ sub-block of $H$ provided that

$$
q^{r}>\sum_{i=0}^{e-1}\left(\begin{array}{c}
j-1 \\
i
\end{array}\right)(q-1)^{i} \times \sum_{s=0}^{l-1}\left(\begin{array}{c}
m-1 \\
s
\end{array}\right)\left(\sum_{i=1}^{e}\left(\begin{array}{l}
t \\
i
\end{array}\right)(q-1)^{i}\right)^{s}
$$

The proof is completed by replacing $j$ by $t$.

Example 2.3. Consider a $(16,9)$ binary code with the $7 \times 16$ matrix $H$ which is constructed by the synthesis procedure explained in the proof of Theorem 2.2 by considering $m=4, t=4, l=2, e=2, q=2$. 


$$
H=\left[\begin{array}{llllllllllllllll}
1 & 0 & 0 & 1 & 0 & 0 & 0 & 0 & 0 & 0 & 0 & 0 & 1 & 0 & 1 & 1 \\
0 & 1 & 1 & 1 & 0 & 0 & 0 & 0 & 0 & 0 & 1 & 0 & 0 & 1 & 1 & 0 \\
0 & 0 & 1 & 1 & 0 & 0 & 0 & 0 & 0 & 1 & 0 & 0 & 1 & 0 & 1 & 0 \\
0 & 0 & 0 & 0 & 1 & 0 & 0 & 1 & 1 & 0 & 0 & 0 & 0 & 1 & 1 & 0 \\
0 & 0 & 0 & 0 & 0 & 1 & 1 & 1 & 0 & 0 & 0 & 0 & 1 & 0 & 1 & 0 \\
0 & 0 & 0 & 0 & 0 & 0 & 1 & 1 & 0 & 0 & 1 & 0 & 0 & 1 & 1 & 0 \\
0 & 0 & 0 & 0 & 0 & 0 & 0 & 0 & 1 & 1 & 0 & 1 & 1 & 1 & 1 & 1
\end{array}\right]
$$

The above code can locate 2 or less corrupted sub-blocks each containing 2 or less errors because all the syndromes of such error are non-zero which can be easily verified.

\section{Location of $e$ or less errors in multiple sub-blocks}

This section is devoted for obtaining lower and upper bounds on the number of check digits required for the existence of a linear code over $G F(q)$ capable of locating multiple corrupted sub-blocks each containing $e$ or less errors. Firstly, we obtain the lower bound on the number of check digits for such a linear code and we follow similar approach used in Theorem 1 of [17].

Theorem 3.1. An $(n, k)$ linear code over $G F(q)$ which is divided into $m$ mutually exclusive sub-blocks of length $t$ each, is capable of locating any $l(\leq m)$ or less corrupted sub-blocks each containing $e$ or less errors. The number of parity check digits $r$ needed for such an EL-code is bounded below by

$$
r \geq \log _{q}\left[1+\sum_{j=1}^{l}\left[\left(\begin{array}{c}
m \\
j
\end{array}\right)\left\{\sum_{i=1}^{\lfloor e / 2\rfloor}\left(\begin{array}{l}
t \\
i
\end{array}\right)(q-1)^{i}\right\}^{j}\right]\right] .
$$

( \lfloor\rfloor$\rfloor$ means the greatest integer less than or equal to $x$.)

Proof. In the proof, we first count the number of syndromes needed to be distinct according to conditions $(i)$ and $(i i)$ and then make this number less than or equal to $q^{r}$, maximum possible number of distinct syndromes.

For any $j \leq l$, any syndrome produced by $j\lfloor e / 2\rfloor$ or less errors occurring in any $j$ sub-blocks with a sub-block containing not more than $\lfloor e / 2\rfloor$ errors must be distinct from any such syndrome likewise resulting from such 
$j\lfloor e / 2\rfloor$ or less errors in the same set of $j$ sub-blocks, according to condition $(i)$. The number of distinct syndromes is given by (2.1) i.e.

$$
\left\{\sum_{i=1}^{\lfloor e / 2\rfloor}\left(\begin{array}{l}
t \\
i
\end{array}\right)(q-1)^{i}\right\}^{j}
$$

Further, syndromes resulted by combinations of up to le errors in different sets of $l$ or less sub-blocks each not containing more that $e$ errors must be distinct by condition $(i i)$; as a result, syndromes of up to $j\lfloor e / 2\rfloor$ errors, whether confined to the same $j$ sub-blocks or to different such subblocks will be all distinct. As the number of ways in which we can choose $j$ sub-blocks from $m$ sub-blocks is $\left(\begin{array}{c}m \\ j\end{array}\right)$, so the number of distinct syndromes excluding zero syndrome is

$$
\sum_{j=1}^{l}\left[\left(\begin{array}{c}
m \\
j
\end{array}\right)\left\{\sum_{i=1}^{\lfloor e / 2\rfloor}\left(\begin{array}{l}
t \\
i
\end{array}\right)(q-1)^{i}\right\}^{j}\right]
$$

Hence

$$
q^{r} \geq 1+\sum_{j=1}^{l}\left[\left(\begin{array}{c}
m \\
j
\end{array}\right)\left\{\sum_{i=1}^{\lfloor e / 2\rfloor}\left(\begin{array}{l}
t \\
i
\end{array}\right)(q-1)^{i}\right\}^{j}\right]
$$

Remark 3.2. For $l=1$ and $q=2$, the bound 3.2 coincides with Theorem 1 by Wolf and Elpsas [17].

In the following result, an upper bound on the number of check digits needed for the existence of a code considered in Theorem 3.1 is derived. Similar technique of Theorem 2.2 is used for the proof.

Theorem 3.3. An $(n, k)$ linear EL-code over $G F(q)$ which is divided into $m$ mutually exclusive sub-blocks of length $t$ each, is capable of locating any $l(\leq m / 2)$ or less corrupted sub-blocks each containing $e$ or less errors. There shall always exist such an EL-code having $r$ parity check digits provided that

$$
q^{r}>\sum_{i=0}^{e-1}\left(\begin{array}{c}
t-1 \\
i
\end{array}\right)(q-1)^{i} \times \sum_{s=0}^{2 l-1}\left(\begin{array}{c}
m-1 \\
s
\end{array}\right)\left(\sum_{i=1}^{e}\left(\begin{array}{l}
t \\
i
\end{array}\right)(q-1)^{i}\right)^{s}
$$


Proof. For construction of a suitable $(n-k) \times n$ parity check matrix $H$ for the required code, we first assume that the columns of the first $m-1$ sub-blocks of $H$ and the first $j-1$ columns $h_{1}, h_{2}, \ldots, h_{j-1}$ of the $m^{t h}$ sub-block are suitably added. We now put down the conditions to add $j^{\text {th }}$ column $h_{j}$ of the $m^{\text {th }}$ sub-block of the matrix $H$ as follows:

According to condition $(i)$, the syndrome getting from the occurrence of $e$ or less errors occurring in any $l$ or less sub-blocks must be non-zero. So, $h_{j}$ should not be a linear combination of previous any $e-1$ or less columns of the $m^{\text {th }}$ sub-block, along with any $(l-1) e$ or less columns among the previous any $l-1$ or less sub-blocks having not more than $e$ columns within a sub-block. Therefore, the number of linear combinations of columns that $h_{j}$ can not be equal to is given by $(2.4)$, i.e.

$$
\sum_{i=0}^{e-1}\left(\begin{array}{c}
j-1 \\
i
\end{array}\right)(q-1)^{i} \times \sum_{s=0}^{l-1}\left(\begin{array}{c}
m-1 \\
s
\end{array}\right)\left\{\sum_{i=1}^{e}\left(\begin{array}{l}
t \\
i
\end{array}\right)(q-1)^{i}\right\}^{s}
$$

Further, according to condition $(i i)$, the syndrome getting from $e$ or less errors occurring in any $l$ or less sub-blocks must be different from the syndrome getting from $e$ or less errors occurring in any other set of $l$ or less sub-blocks (one sub-block contains not more than $e$ errors). In view of this, $h_{j}$ should not be a linear combination of previous any $e-1$ or less columns of the $m^{\text {th }}$ sub-block, together with any $e(2 l-1)$ or less columns amongst the previous any $2 l-1$ or less sub-blocks such that maximum number of columns in a sub-block is $e$. In other words, $h_{j}$ can be added to the $m^{t h}$ sub-block of $H$ on the condition that

$$
\begin{aligned}
& \quad \mathrm{h}_{j} \neq v_{b_{1}} h_{b_{1}}+v_{b_{2}} h_{b_{2}}+\ldots+v_{b_{e}-1} h_{b_{e-1}} \\
& +u_{w_{11}} h_{w_{11}}+u_{w_{12}} h_{w_{12}}+\ldots+u_{w_{1 e}} h_{w_{1 e}} \\
& +u_{w_{21}} h_{w_{21}}+u_{w_{22}} h_{w_{22}}+\ldots+u_{w_{2 e}} h_{w_{2 e}} \\
& +\ldots \ldots \ldots \ldots \\
& +\ldots \ldots \ldots \ldots . . \\
& +u_{w_{s 1}} h_{w_{s 1}}+u_{w_{s 2}} h_{w_{s 2}}+\ldots+u_{w_{s e}} h_{w_{s e}}
\end{aligned}
$$


where $v_{b_{j}}, u_{w_{i j}} \in G F(q), s \leq 2 l-1$ and $h_{b_{j}}$ 's are any $e-1$ columns from amongst the columns $h_{1}, h_{2}, \ldots, h_{j-1}$ of the $m^{t h}$ sub-block and $h_{w_{i j}}$ 's are any $e$ columns within a sub-block (for same value of $i$ ) chosen from previous $m-1$ sub-blocks.

Thus, the number of linear combinations on the R.H.S. of (3.5) is

$$
\sum_{i=0}^{e-1}\left(\begin{array}{c}
j-1 \\
i
\end{array}\right)(q-1)^{i} \times \sum_{s=0}^{2 l-1}\left(\begin{array}{c}
m-1 \\
s
\end{array}\right)\left(\sum_{i=1}^{e}\left(\begin{array}{l}
t \\
i
\end{array}\right)(q-1)^{i}\right)^{s}
$$

Note that number of linear combinations computed in (3.4) is also considered in (3.6).

Therefore, $h_{j}$ can be added to the $m^{\text {th }}$ sub-block of $H$ provided that

$$
q^{r}>\sum_{i=0}^{e-1}\left(\begin{array}{c}
j-1 \\
i
\end{array}\right)(q-1)^{i} \times \sum_{s=0}^{2 l-1}\left(\begin{array}{c}
m-1 \\
s
\end{array}\right)\left(\sum_{i=1}^{e}\left(\begin{array}{l}
t \\
i
\end{array}\right)(q-1)^{i}\right)^{s}
$$

Putting $t$ in place of $j$ gives the theorem.

Remark 3.4. For $l=1, q=2$, the bound (3.3) reduces to

$$
\begin{array}{r}
2^{r}>\sum_{i=0}^{e-1}\left(\begin{array}{c}
t-1 \\
i
\end{array}\right) \times\left\{1+(m-1) \sum_{i=1}^{e}\left(\begin{array}{l}
t \\
i
\end{array}\right)\right\} \\
\Longrightarrow 2^{r}>1+\sum_{i=1}^{e-1}\left(\begin{array}{c}
t-1 \\
i
\end{array}\right)+(m-1) \sum_{i=1}^{e}\left(\begin{array}{c}
t \\
i
\end{array}\right) \sum_{i=0}^{e-1}\left(\begin{array}{c}
t-1 \\
i
\end{array}\right) \\
\Longrightarrow 2^{r} \geq 2+\sum_{i=1}^{e-1}\left(\begin{array}{c}
t-1 \\
i
\end{array}\right)+(m-1) \sum_{i=1}^{e}\left(\begin{array}{c}
t \\
i
\end{array}\right)\left\{1+\sum_{i=1}^{e-1}\left(\begin{array}{c}
t-1 \\
i
\end{array}\right)\right\} .
\end{array}
$$

This coincides with the result obtained by Dass and Muttoo [7], an improvement of Theorem 2, Wolf and Elpsas [17].

Example 3.5. Consider a $(16,3)$ binary code with the $13 \times 16$ matrix $H$ which is constructed by the synthesis procedure discussed in the proof of 
Theorem 3.3 by considering $m=4, t=4, l=2, e=2, q=2$.

$$
H=\left[\begin{array}{llllllllllllllll}
1 & 0 & 0 & 1 & 0 & 0 & 0 & 0 & 0 & 0 & 0 & 0 & 0 & 0 & 0 & 0 \\
0 & 1 & 1 & 1 & 0 & 0 & 0 & 0 & 0 & 0 & 0 & 0 & 0 & 0 & 0 & 0 \\
0 & 0 & 1 & 1 & 0 & 0 & 0 & 0 & 0 & 0 & 0 & 0 & 0 & 0 & 0 & 0 \\
0 & 0 & 0 & 0 & 1 & 0 & 0 & 1 & 0 & 0 & 0 & 0 & 0 & 0 & 0 & 0 \\
0 & 0 & 0 & 0 & 0 & 1 & 1 & 1 & 0 & 0 & 0 & 0 & 0 & 0 & 0 & 0 \\
0 & 0 & 0 & 0 & 0 & 0 & 1 & 1 & 0 & 0 & 0 & 0 & 0 & 0 & 0 & 0 \\
0 & 0 & 0 & 0 & 0 & 0 & 0 & 0 & 1 & 0 & 0 & 1 & 0 & 0 & 0 & 0 \\
0 & 0 & 0 & 0 & 0 & 0 & 0 & 0 & 0 & 1 & 1 & 1 & 0 & 0 & 0 & 0 \\
0 & 0 & 0 & 0 & 0 & 0 & 0 & 0 & 0 & 0 & 1 & 1 & 0 & 0 & 0 & 0 \\
0 & 0 & 0 & 0 & 0 & 0 & 0 & 0 & 0 & 0 & 0 & 0 & 1 & 0 & 0 & 1 \\
0 & 0 & 0 & 0 & 0 & 0 & 0 & 0 & 0 & 0 & 0 & 0 & 0 & 1 & 1 & 1 \\
0 & 0 & 0 & 0 & 0 & 0 & 0 & 0 & 0 & 0 & 0 & 0 & 0 & 0 & 1 & 1 \\
1 & 1 & 1 & 1 & 1 & 1 & 1 & 1 & 1 & 1 & 1 & 1 & 1 & 1 & 1 & 1
\end{array}\right]
$$

The above $(16,3)$ code can locate 2 or less corrupted sub-blocks each containing 2 or less errors. We can easily verify that all the syndromes of errors are non-zero and distinct in different sets of 2 or less sub-blocks.

\section{Correction of $e$ or fewer errors in multiple sub-blocks}

In this section, we extend the study of Section 2 and consider the correction of errors occuring in multiple sub-blocks. We present here an upper bound on the number of parity check digits for the existence of a linear code that is capable of correction of $e$ or less errors occurring in multiple sub-blocks. The lower bound on the number of parity check digits of such codes is already obtained in [10] (Theorem 3.4) which may be stated as follows.

Theorem 4.1. For given $l$ and $e$, suppose that an $(n, k)$ linear code over $G F(q)$ subdivided into $m$ mutually exclusive sub-blocks of length $t$ each, that corrects $e$ or less errors occurring in any $l$ or less sub-blocks. The number of parity check digits $r$ for such an $(n, k)$ linear code is bounded below by

$$
q^{r} \geq 1+\sum_{j=1}^{l}\left[\left(\begin{array}{c}
m \\
j
\end{array}\right)\left\{\sum_{i=1}^{e}\left(\begin{array}{l}
t \\
i
\end{array}\right)(q-1)^{i}\right\}^{j}\right] .
$$

Theorem 4.2. For given $l$ and $e$, suppose that an $(n, k)$ linear code over $G F(q)$ whose is divided into $m$ mutually exclusive sub-blocks of length $t$ 
each, corrects $e$ or less errors occurring in any $l$ or less sub-blocks $(l \leq$ $m / 2, e \leq t / 2)$. There shall always exist such an $(n, k)$ linear code having $r$ parity check digits provided that

$$
\begin{aligned}
q^{r} & >\sum_{i=0}^{e-1}\left(\begin{array}{c}
j-1 \\
i
\end{array}\right)(q-1)^{i} \times \sum_{s=l}^{2 l-1}\left(\begin{array}{c}
m-1 \\
s
\end{array}\right)\left(\sum_{i=1}^{e}\left(\begin{array}{l}
t \\
i
\end{array}\right)(q-1)^{i}\right)^{s} \\
& +\sum_{i=0}^{2 e-1}\left(\begin{array}{c}
j-1 \\
i
\end{array}\right)(q-1)^{i} \times \sum_{s=0}^{l-1}\left(\begin{array}{c}
m-1 \\
s
\end{array}\right)\left(\sum_{i=1}^{2 e}\left(\begin{array}{c}
t \\
i
\end{array}\right)(q-1)^{i}\right)^{s} .
\end{aligned}
$$

Proof. The result is also proved by the same techenique used in Theorem 2.2 and Theorem 3.3. Suppose that the columns of the first $m-1$ sub-blocks of $H$ and the first $j-1$ columns $h_{1}, h_{2}, \ldots, h_{j-1}$ of the $m^{\text {th }}$ subblock have been added appropriately. To add the $j^{\text {th }}$ column $h_{j}$ of the $m^{\text {th }}$ sub-block of the matrix $H$, we need to follow the conditions $(i)-(i i i)$.

According to conditions $(i)-(i i)$, the syndrome of $e$ or less errors occurring in any $l$ or less sub-blocks must be non-zero and different from the syndrome getting from $e$ or less errors in any other set of $l$ or less subblocks. So, the column $h_{j}$ can be added to the $m^{\text {th }}$ sub-block provided that it should not be any one of the linear combinations of the R.H.S. of (3.5) and the number of such linear combinations is given by (3.6), i.e.

$$
\sum_{i=0}^{e-1}\left(\begin{array}{c}
j-1 \\
i
\end{array}\right)(q-1)^{i} \times \sum_{s=0}^{2 l-1}\left(\begin{array}{c}
m-1 \\
s
\end{array}\right)\left(\sum_{i=1}^{e}\left(\begin{array}{l}
t \\
i
\end{array}\right)(q-1)^{i}\right)^{s} .
$$

Further, according to condition ( $i i i)$, the syndrome of $e$ or less errors occurring in any $l$ or less sub-blocks must be different from the syndrome getting from $e$ or less errors in any same set of $l$ or less sub-blocks. In view of this, $h_{j}$ should not be a linear combination of previous any $2 e-1$ or less columns of the $m^{\text {th }}$ sub-block, together with any $2 e(l-1)$ or less columns amongst any previous $l-1$ or less sub-blocks such that maximum number of columns in a sub-block is $2 e$. In other words, for adding the column $h_{j}$ to the $m^{t h}$ sub-block of $H$, the condition is 


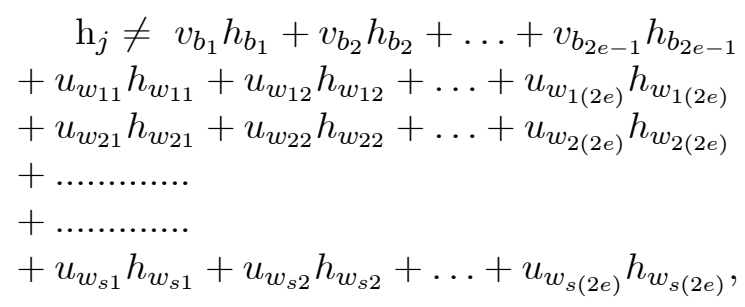

where $v_{b_{j}}, u_{w_{i j}} \in G F(q), s \leq l-1$ and $h_{b_{j}}$ 's are any $2 e-1$ columns from amongst the columns $h_{1}, h_{2}, \ldots, h_{j-1}$ of the $m^{\text {th }}$ sub-block and $h_{w_{i j}}$ 's are any $2 e$ columns within a sub-block (for same value of $i$ ) chosen from previous $m-1$ sub-blocks.

The number of linear combinations on the R.H.S. of (4.2), including the vector of all zeros, can be counted as

$$
\sum_{i=0}^{2 e-1}\left(\begin{array}{c}
j-1 \\
i
\end{array}\right)(q-1)^{i} \times \sum_{s=0}^{l-1}\left(\begin{array}{c}
m-1 \\
s
\end{array}\right)\left(\sum_{i=1}^{2 e}\left(\begin{array}{l}
t \\
i
\end{array}\right)(q-1)^{i}\right)^{s} .
$$

We note that some linear combinations on the R.H.S. of (4.2) are already considered in (3.5). The number of common linear combinations is

$$
\sum_{i=0}^{e-1}\left(\begin{array}{c}
j-1 \\
i
\end{array}\right)(q-1)^{i} \times \sum_{s=0}^{l-1}\left(\begin{array}{c}
m-1 \\
s
\end{array}\right)\left(\sum_{i=1}^{e}\left(\begin{array}{l}
t \\
i
\end{array}\right)(q-1)^{i}\right)^{s} .
$$

Therefore, the total number of linear combinations that $h_{j}$ can not be is the sum of number computed in (3.6) and in (4.3) minus the number computed in (4.4). These all combinations might yield distinct sums. Thus, we can add the column $h_{j}$ to the $m^{t h}$ sub-block of $H$ provided

$$
\begin{aligned}
q^{r} & >\sum_{i=0}^{e-1}\left(\begin{array}{c}
j-1 \\
i
\end{array}\right)(q-1)^{i} \times \sum_{s=0}^{2 l-1}\left(\begin{array}{c}
m-1 \\
s
\end{array}\right)\left(\sum_{i=1}^{e}\left(\begin{array}{l}
t \\
i
\end{array}\right)(q-1)^{i}\right)^{s} \\
& +\sum_{i=0}^{2 e-1}\left(\begin{array}{c}
j-1 \\
i
\end{array}\right)(q-1)^{i} \times \sum_{s=0}^{l-1}\left(\begin{array}{c}
m-1 \\
s
\end{array}\right)\left(\sum_{i=1}^{2 e}\left(\begin{array}{l}
t \\
i
\end{array}\right)(q-1)^{i}\right)^{s} \\
& -\sum_{i=0}^{e-1}\left(\begin{array}{c}
j-1 \\
i
\end{array}\right)(q-1)^{i} \times \sum_{s=0}^{l-1}\left(\begin{array}{c}
m-1 \\
s
\end{array}\right)\left(\sum_{i=1}^{e}\left(\begin{array}{c}
t \\
i
\end{array}\right)(q-1)^{i}\right)^{s} .
\end{aligned}
$$


or

$$
\begin{aligned}
(4.5) q^{r} & >\sum_{i=0}^{e-1}\left(\begin{array}{c}
j-1 \\
i
\end{array}\right)(q-1)^{i} \times \sum_{s=l}^{2 l-1}\left(\begin{array}{c}
m-1 \\
s
\end{array}\right)\left(\sum_{i=1}^{e}\left(\begin{array}{l}
t \\
i
\end{array}\right)(q-1)^{i}\right)^{s} \\
& +\sum_{i=0}^{2 e-1}\left(\begin{array}{c}
j-1 \\
i
\end{array}\right)(q-1)^{i} \times \sum_{s=0}^{l-1}\left(\begin{array}{c}
m-1 \\
s
\end{array}\right)\left(\sum_{i=1}^{2 e}\left(\begin{array}{l}
t \\
i
\end{array}\right)(q-1)^{i}\right)^{s} .
\end{aligned}
$$

The theorem follows by replacing $j$ by $t$ in (4.5).

Example 4.3. Consider a $(20,5)$ binary code with the $15 \times 20$ matrix $H$ which is constructed by the synthesis procedure considered in the proof of Theorem 4.2 by taking $m=5, t=4, l=2, e=2, q=2$.

$H=\left[\begin{array}{llllllllllllllllllll}1 & 0 & 0 & 0 & 0 & 0 & 0 & 0 & 0 & 0 & 0 & 0 & 0 & 0 & 0 & 1 & 1 & 0 & 0 & 0 \\ 0 & 1 & 0 & 0 & 0 & 0 & 0 & 0 & 0 & 0 & 0 & 0 & 0 & 0 & 0 & 1 & 0 & 1 & 0 & 0 \\ 0 & 0 & 1 & 0 & 0 & 0 & 0 & 0 & 0 & 0 & 0 & 0 & 0 & 0 & 0 & 1 & 0 & 0 & 1 & 0 \\ 0 & 0 & 0 & 1 & 0 & 0 & 0 & 0 & 0 & 0 & 0 & 0 & 0 & 0 & 0 & 1 & 0 & 0 & 0 & 1 \\ 0 & 0 & 0 & 0 & 1 & 0 & 0 & 0 & 0 & 0 & 0 & 0 & 0 & 0 & 0 & 1 & 1 & 0 & 0 & 0 \\ 0 & 0 & 0 & 0 & 0 & 1 & 0 & 0 & 0 & 0 & 0 & 0 & 0 & 0 & 0 & 1 & 0 & 1 & 0 & 0 \\ 0 & 0 & 0 & 0 & 0 & 0 & 1 & 0 & 0 & 0 & 0 & 0 & 0 & 0 & 0 & 1 & 0 & 0 & 1 & 0 \\ 0 & 0 & 0 & 0 & 0 & 0 & 0 & 1 & 0 & 0 & 0 & 0 & 0 & 0 & 0 & 1 & 0 & 0 & 0 & 1 \\ 0 & 0 & 0 & 0 & 0 & 0 & 0 & 0 & 1 & 0 & 0 & 0 & 0 & 0 & 0 & 1 & 1 & 0 & 0 & 0 \\ 0 & 0 & 0 & 0 & 0 & 0 & 0 & 0 & 0 & 1 & 0 & 0 & 0 & 0 & 0 & 1 & 0 & 1 & 0 & 0 \\ 0 & 0 & 0 & 0 & 0 & 0 & 0 & 0 & 0 & 0 & 1 & 0 & 0 & 0 & 0 & 1 & 0 & 0 & 1 & 0 \\ 0 & 0 & 0 & 0 & 0 & 0 & 0 & 0 & 0 & 0 & 0 & 1 & 0 & 0 & 0 & 1 & 0 & 0 & 0 & 1 \\ 0 & 0 & 0 & 0 & 0 & 0 & 0 & 0 & 0 & 0 & 0 & 0 & 1 & 0 & 0 & 1 & 1 & 0 & 0 & 1 \\ 0 & 0 & 0 & 0 & 0 & 0 & 0 & 0 & 0 & 0 & 0 & 0 & 0 & 1 & 0 & 1 & 0 & 1 & 0 & 0 \\ 0 & 0 & 0 & 0 & 0 & 0 & 0 & 0 & 0 & 0 & 0 & 0 & 0 & 0 & 1 & 1 & 0 & 0 & 1 & 1 \\ & & & & & & & & & & & & & & & & & & & \end{array}\right]$

The above code can correct 2 or less errors occurring in any 2 or less sub-blocks. It can be also checked that all the syndromes of 2 or less errors in any 2 or less sub-blocks are non-zero and distinct whether in the same set of 2 or less sub-blocks or different sets of 2 or less sub-blocks. It may be noted that in Example 4.3, the syndromes may coincide in the same set of 2 or less sub-blocks, but they need to be distinct in different sets of 2 or less sub-blocks. 
Remark 4.4. The distance of the $(20,5)$ binary code in the above example is 5 , but it is capable of correcting 4 or less errors confined to any 2 subblocks, maximum number of errors in one sub-block is 2 .

\section{References}

[1] P. K. Das, Codes Detecting and Locating Solid Burst Errors, Romanian Journal of Mathematics and Computer Science, Vol. 2 (2), pp. 1-10, (2012).

[2] P. K. Das and L. K. Vashisht, Error Locating Codes By Using Blockwise-Tensor Product of Blockwise Detecting/Correcting Codes, Khayyam Journal of Mathematics, Vol. 2 (1), pp. 6-17, (2016).

[3] P. K. Das, Location of Multiple Sub-Blocks with Burst Errors, Electronic Notes in Discrete Mathematics, Vol. 63, pp. 117-123, (2017).

[4] B. K. Dass, Burst error locating linear codes, J. Inf. and Optimization Sciences, Vol. 3(1), pp. 77-80, (1982).

[5] B. K. Dass and S. Madan, Repeated Burst Error Locating Linear Codes, Discrete Mathematics, Algorithms and Applications, Vol. 2 (2), pp. 181188, (2010).

[6] B. K. Dass and S. Madan, Blockwise Repeated Burst Error Correcting Linear Codes, Ratio Mathematica -Journal of Applied Mathematics, Vol. 20, pp. 97-126 (2010).

[7] B. K. Dass and S. K. Muttoo, Comments on Error-locating Codes A New Concept in Error Control, IEEE Transactions on Information Theory, Vol. 27 (1), 136, (1981).

[8] B. K. Dass and V. Tyagi, Bounds on blockwise burst error correcting linear codes, Information Sciences, vol. 20 (3), pp. 167-164, (1980).

[9] E. Fujiwara and M. Kitakami, A Class of Error-Locating Codes for Byte-Organized Memory Systems, IEEE Transactions on Information Theory, vol. 40 (6), pp. 1857-1865, (Nov. 1994). 
[10] W. H. Fong, Coding Bounds for Multiple Phased-Burst Correction and Single Burst Correction Codes, http://arxiv.org/pdf/1104.1408.pdf, (2011).

[11] M. Kitakami and S. Jiang, Code Design and Decoding Methods for Burst Error Locating Codes, Proceedings of the 11th Pacific Rim International Symposium on Dependable Computing, (2005), (doi: 10.1109/PRDC.2005.24).

[12] M. Kitakami, S. Jiang and E. Fujiwara, Metrics of error locating codes, IEICE Trans. Fundamentals of Electronics, Communications and Computer, vol. E80-A(11), pp. 2117-2122 (1997).

[13] W. W. Peterson and E. J. Weldon(Jr.), Error-Correcting Codes, 2nd edition, The MIT Press, Mass, (1972).

[14] G. E. Sacks, Multiple error correction by means of parity-checks, IRE Trans. Inform. Theory, IT-4, pp. 145-147 (1958).

[15] J. K. Wolf, On an extended class of error-locating codes, Information and Control, vol. 8, pp. 163-169 (1965).

[16] J. K. Wolf, On Codes Derivable from the Tensor Product of Check Matrices, IEEE Trans. on Inform. Theory, vol. 11 (2), pp. 281-284 (1965)

[17] J. K. Wolf and B. Elspas, Error-locating codes-a new concept in error control. IEEE Trans. Inform. Theory IT-9, pp. 20-28, (1963).

\section{Pankaj Kumar Das}

Department of Mathematical Sciences, Tezpur University, Napaam, Sonitpur, Assam-784028, India

e-mail : pankaj4@tezu.ernet.in 